\title{
Surgical reconstruction of the sternum following cardiac surgery complicated by mediastinitis -implantation of a custom-made sternal implant: a case report
}

\author{
Jason Trevis ${ }^{1}$, Joel Dunning ${ }^{2}$, James $\mathrm{McVie}^{3}$ \\ ${ }^{1}$ Newcastle University Medical School, Newcastle upon Tyne NE2 4HH, UK; ${ }^{2}$ Consultant Thoracic Surgeon, James Cook University Hospital, \\ Middlesbrough, UK; ${ }^{3}$ Consultant Orthopaedic Surgeon, James Cook University Hospital, Middlesbrough, UK \\ Correspondence to: Jason Trevis. Newcastle Medical School, Newcastle University, Framlington Place, Newcastle-upon-Tyne, NE2 4HH, UK. \\ Email: Jason.trevis@hotmail.com.
}

\begin{abstract}
Mediastinitis represents an uncommon but potentially life-threatening complications following median sternotomy. There remains a lack of consensus regarding the optimal treatment option(s). Custom tridimensional titanium printing provides an effective method for construction of complex prothesis. We report the case of a 49-year-old female with previous sternal dehiscence and deep wound infection following coronary artery bypass grafts, necessitating sternectomy. A year later, she presented with chronic severe pain and audible crepitations of her ribs/clavicles. Lack of identification of a mechanical cause, poor localisation and suboptimal medical pain management culminated in the consideration for a novel approach for sternal reconstruction. By way of bridging the final placement of the titanium prothesis, the patient underwent placement of a methyl methacrylate spacer. The surgical approach involved the removal of the temporary spacer, with tissue samples taken to guide post-operative antimicrobial therapy. Concurrently, the reaming, irrigation and aspiration technique of bone marrow harvesting, for seeding of the central web structure of the implant, was employed under fluoroscopic guidance. Following adequate dissection under the pectoral muscle, and debridement of the anterior chest wall, final placement of the prothesis was made via bicortical fixation to the ribs. Finally, the mobilised pectoral muscles were sutured medially and fixed to the previously placed rectus abdominis flap. Three-dimensional printing of a custom prosthesis offers a novel and effective approach to full reconstruction; maintaining pulmonary mechanics and stability of the anterior chest wall.
\end{abstract}

Keywords: Mediastinitis; sternal reconstruction; custom prosthesis; cardiac surgery; case report

Received: 15 January 2020; Accepted: 05 February 2020; Published: 25 August 2020.

doi: $10.21037 /$ ccts.2020.02.01

View this article at: http://dx.doi.org/10.21037/ccts.2020.02.01

\section{Introduction}

Mediastinitis represents a relatively uncommon but potentially life-threatening complication following a median sternotomy, with incidences ranging from $0.25-5 \%$ (1). It may result from direct haematogenous spread of bacteria or from the extension of infection from adjacent structures e.g., oesophagus, airways, lungs or head and neck (descending necrotising infection). Deep sternal wound infections by definition progress under the sternum and the anterior mediastinum, with a range of presentations from stable to critically ill. Diagnostic criteria has been described by the Centres for Disease Control and Prevention (CDC) (2) with signs and symptoms typically presenting within 30 days of cardiac surgery; whilst the predominant risk factors for its development include diabetes mellitus and obesity.

\section{Case presentation (Figure 1)}

A 49-year-old female, with a history of type 2 diabetes and associated neuropathy, presented requiring full sternal reconstruction due to complications from coronary artery 

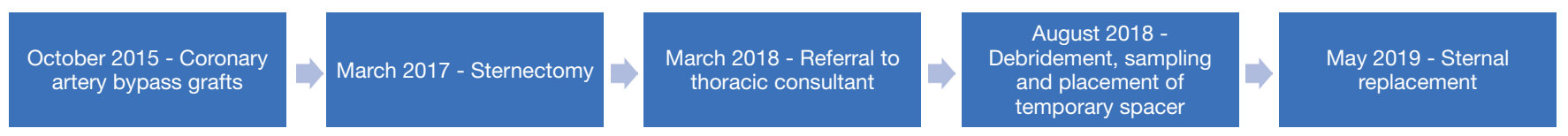

Figure 1 Summary of the key events during the patient's care journey.

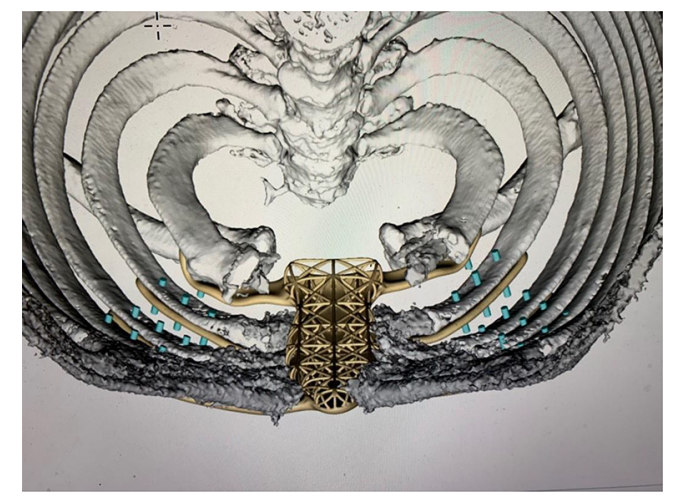

Figure 2 Computer-generated reconstruction during the design phase.

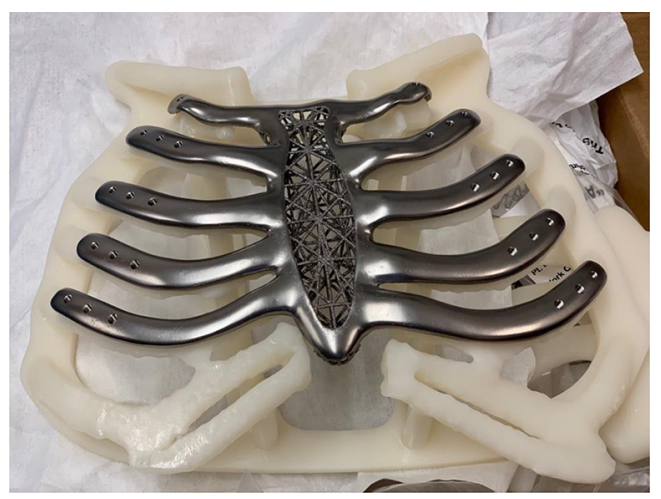

Figure 3 Custom prosthesis designed by $4 \mathrm{Web}$.

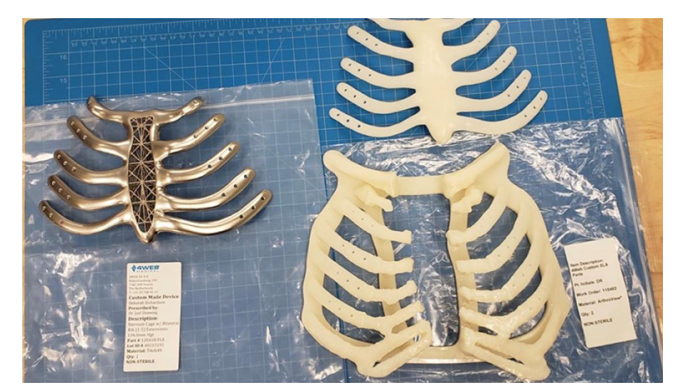

Figure 4 Titanium prosthesis (left), plastic reconstructions for preoperative planning (right). bypass graft (CABG) surgery. In 2015 she underwent triple vessel CABGs, re-presenting two weeks later with sternal wound dehiscence and deep wound infection necessitating repair with sternectomy (Figure 1). Debridement resulted in the removal of most of her sternum, requiring reconstruction using a right rectus abdominis muscle flap.

A year later, following a bout of sneezing, she reported a 'popping' sensation predominantly in her right rib cage; $\mathrm{X}$-ray showed no obvious breakage or lung pathology. She developed chronic severe pain and audible crepitations of her ribs/clavicles, confounded by post traumatic stress disorder from previous surgery. Due to the lack of identification of a mechanical cause, and poor localisation, intercostal nerve blockade through cryoanalgesia was not feasible; therefore medical management was initiated (Zomorph and Pregabalin).

Due to suboptimal pain control, the patient became wheelchair-bound, with diminishing quality of life. Awareness of a novel management option in April 2018 led to the consideration for sternal reconstruction. The patient initially underwent debridement of sternum and placement of a temporary methyl methacrylate spacer; bridging the gap for the insertion of the custom titanium prosthesis. As a result of this debridement, tissue cultures returned the following:

(I) Heavy growth of Klebsiella pneumoniae.

(II) Heavy growth of Streptococcus vestibularis.

(III) Heavy growth of Streptococcus oralis.

\section{Patient specific implant}

The patient's CT images were used by 4 WEB in order to create the custom prosthesis, with input from the lead surgeons to adjust for specific intraoperative details, these included; fixation technique to the ribs and design of sternal body with a lightweight web structure. As a result, three custom implants were 3D printed (Figures 2-4);

- The final titanium implant.

- Plastic replica of the implant and anterior chest wall to be used for preoperative planning, and intraoperative guidance. 


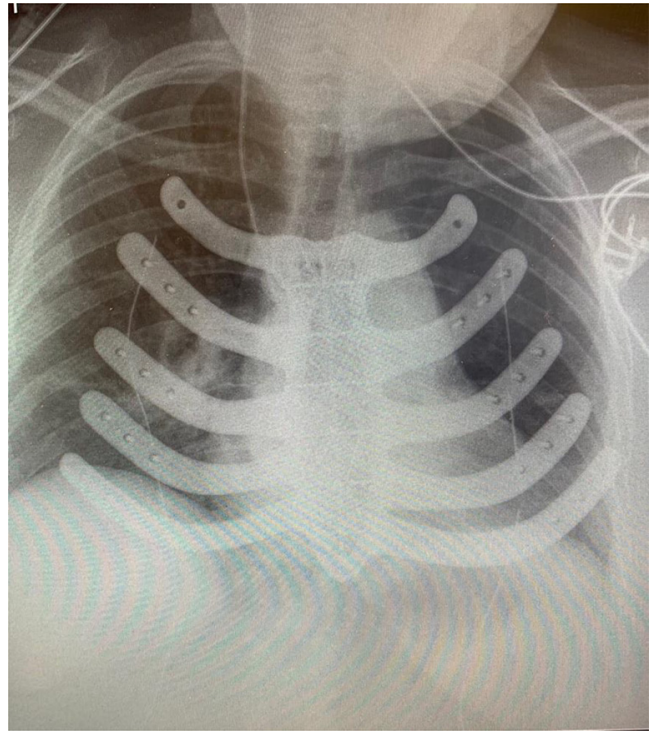

Figure 5 Post-operative chest X-ray.

The implant combines the reconstruction concepts described above, utilising bone marrow from the femur to promote osteogenesis, whilst providing anterior chest wall stability; particularly in patients with little sternal tissue remaining.

\section{Surgical approach}

The procedure involved both orthopaedic and cardiothoracic surgical input. Proceeding with the reopening of the previous sternotomy scar with dissection of the pectoral muscles off the anterior chest wall, to allow for removal of the temporary implant. During which the plastic implant replica was utilised as a guide for dissection margins in preparation for final implantation.

Concurrently, bone marrow was harvested from the left femur, using the reaming, irrigation and aspiration (RIA) technique, under fluoroscopic guidance. The marrow was later utilised to seed the central web structure of the implant, along with Cerament G (Bonesupport, Sweden) pellets. Cerament $\mathrm{G}$ is a gentamicin-loaded calcium sulphate/hydroxyapatite biocomposite. The gentamicin elution is reliable and consistent and has a high initial peak that remains above the minimum inhibitory concentration (MIC) for gentamicin sensitive Staphlococcus Aureus and Pseudomonas aeruginosa for at least 28 days in in vitro studies (3).

Following removal of the temporary methyl methacrylate spacer, six tissue samples were taken from the remaining sternum/surrounding tissue and sent for culture and sensitivity. The samples are taken using clean forceps and blade for each and if 3 or more are subsequently positive for bacterial growth, infection is still present. Our patient had 3 positive cultures for Staphlococcus epidermidis and therefore our Infectious Diseases Department was involved to start the appropriate antibiotic regime.

Final placement of the titanium prosthesis was trialled following adequate debridement utilising the guide prosthesis. Minor adjustments were subsequently made in order to bed the prosthesis down sufficiently on the patients' anterior chest wall. Initially pericostal sutures were utilised prior to the placement of 6-8 non-locking screws. Definitive fixation proceeded with locking screws, each sized based upon adequate depth penetrance through the rib, achieving bicortical fixation, whilst minimising the risk of pleural or pericardial damage.

Finally, the mobilised pectoral muscles were sutured medially as a muscle flap, fixing them to the previously placed rectus abdominis flap. Following which, mediastinal, left and right anterior 18 French with handivac chest drains were inserted.

\section{Post-operative follow up}

The patient remained as an inpatient for 7 days following surgery; in order to monitor her post-operative course and maintain appropriate analgesia where necessary. By day 2 post-operatively she was mobilising, and the drains were removed on day 3 (Figure 5). The intra-operative sternal samples demonstrated positive bacterial growth; which subsequently led to the patient being placed on antibiotics for 6 weeks post-operatively.

She was very happy with the improvement of her preoperative pain and her walking distance was improving daily. Unfortunately, she died 3 weeks postoperatively from myocardial infarction due to her underlying cardiac disease.

\section{Ethics and consent}

All procedures performed in this study were in accordance with the ethical standards of the institutional and national research committees and with the Helsinki Declaration (as revised in 2013). Written informed consent was obtained from the patient for publication of this case report. A copy of the written consent is available for review by the editorial office of this journal. 
Table 1 AMSTERDAM (Assiduous Mediastinal Sternal Debridement \& Aimed Management) criteria, van Wingerden et al., Poststernotomy mediastinitis: a classification to initiate and evaluate reconstructive management based on evidence from a structured review, 2014, by permission of the authors and BioMed Central Ltd

\begin{tabular}{|c|c|c|c|c|}
\hline Type & Sternal stability & Bone viability \& stock & Reconstruction & Staging of reconstruction \\
\hline 1 & \multirow[t]{3}{*}{ Stable } & \multirow[t]{3}{*}{ Reasonable } & TNP & (class I, level B) \\
\hline $2 a$ & & & Local muscle flap* & $\begin{array}{l}\text { Primary } \\
\text { (class II, level B) }\end{array}$ \\
\hline $2 b$ & & & Muscle $^{\star \star}$ or Omentum flap & $\begin{array}{l}\text { Delayed } \\
\text { (class I, level B). }\end{array}$ \\
\hline $3 a$ & \multirow{4}{*}{ Unstable } & \multirow{2}{*}{ Viable \& sufficient } & Rewiring/osteosynthesis & $\begin{array}{l}\text { Delayed } \\
\text { (class Ilb, level B) }\end{array}$ \\
\hline $3 b$ & & & Rewiring/osteosynthesis & Primary \\
\hline $4 b$ & & & Omentum flap & (class Ilb, level B) \\
\hline $4 c$ & & & Muscle and Omentum flap & \\
\hline
\end{tabular}

*Always, unilateral or bilateral pectoralis muscle advancement; ${ }^{* \star}$ Frequently, unilateral or bilateral pectoralis muscle advancement; ${ }^{\#}$ Rewiring; ^Osteosynthesis (plates, clips, etc.). TNP, topical negative pressure.

\section{Discussion}

Definitive management options may involve an array of techniques, depending upon the severity of the infection. The general principles of management aim to bring the infection under control, via removal of all infected/ necrotic tissue and foreign material alongside appropriate antimicrobial therapy (4), with subsequent restabilisation of the sternum. Antibiotics remain the initial management option following identification of mediastinitis, with expeditious intravenous (IV) broad spectrum antibiotics recommended. The regimen is subsequently adjusted according to microbial identification and corresponding susceptibilities, as well as dose adjustment for body mass index (BMI) and skeletonised internal mammary grafts, particularly bilateral, to account for reduced sternal and peristernal penetrance $(1,2)$.

Surgical intervention is generally necessary, with wound debridement as a minimum, with more extensive measures employed in a stepwise manor. However, there remains a lack of consensus for optimal surgical management. Generally, two overall closure methods are stated in the literature; primary intention or tertiary intention (delayed primary closure); with subsequent reconstruction if required. Adjuncts to these close techniques include open wound dressings, or closed irrigation with added antibiotics. Topical negative pressure (TNP) therapy may also be used as an adjunct to closure, or as a bridge to surgical management/reconstruction $(2,5)$. The literature suggests that earlier diagnosis and instigation of TNP predicts greater likelihood of survival (2). Conversely, studies have suggested that prolonged TNP can result in chronic infection $(2,5)$.

The timing and type of intervention may be guided via the AMSTERDAM classification as describe by van Wingerden et al. (2) (Table 1).

If sternal instability remains or there is defective thoracic mechanics, fixation may be required to stabilise the anterior chest wall. Many options have been described, utilising an array of prosthetic materials;

(I) Titanium rib plating-optimal fixation is achieved via use of at least three screws each side of the defect, locking bicortically (6).

- The Ley prostheses is a titanium alloy plate designed for stabilisation of the sternum after mediastinitis described in a case report of 3 
patients from Denmark (7).

(II) Methyl methacrylate-typically sandwiched between two layers of mesh for added stability, which can be moulded to the shape of the defect. Disadvantages include increased pain and excessive chest wall ridigity, related to its impermeability to fluids (6).

(III) Prosthetic patches—advantages include the creation of uniform tension at the bone defect edges, whilst usually being well tolerated. Disadvantages include an infection rate range from $10-25 \%$, requiring removal. Despite their effectiveness in thoracic wall defect reconstruction, their use in sternal defects is less so, with the literature suggesting the use of composite implant techniques (6). Generally, patches provide a barrier between the pleural and subcutaneous space while providing a scaffold for regeneration of connective tissue. Patch/mesh types include;

- Synthetic-non-absorbable synthetic woven meshes e.g., polypropylene or polyester and polyetrafluoroethylene (PTFE) (GORE-TEX) and Vicryl mesh.

- Biological e.g., bovine pericardium prosthesis.

- Titanium-providing increased strength, whilst maintaining plasticity and adaptability (6).

(IV) Allograft and autograft - these may be required when there is little bone material to provide support for plates (8). Human and porcine bioprosthetic materials have been described, with advantages including; the ability to incorporate into native tissue with revascularisation and cellular repopulation (6), whilst bone grafts act as a structure for osteoprogenitor cells and bone growth. Examples include-cryopreserved allografts and autografts e.g., en-bloc anterior chest wall and use of the fibula with added titanium plating.

Regardless of the technique used to achieve sternal stability, tissue coverage is most often required to reduce any dead space and providing protection to underlying structures from the exposed sternal parts. Pedicled soft tissue flaps are frequently required to fill the sternal defects or wounds following debridement, as they represent effective reconstruction options due to their highly vascular nature, whilst. Examples include;

(I) Pectoralis major flap.

(II) Transposition of the greater omentum.
(III) Latissimus dorsi flap.

(IV) Rectus abdominis flap.

\section{Conclusions}

Mediastinitis represents an uncommon, but potentially lifethreatening complication following median sternotomy. There remains a lack of consensus regarding the optimal approach for sternal reconstruction, should this be required. The timing and type of intervention may be guided by the AMSTERDAM criteria. However, this does not address the potential need for reconstruction. $3 \mathrm{D}$ printing of a custom prosthesis offers a novel and effective approach to full reconstruction; maintaining pulmonary mechanics and stability of the anterior chest wall. After reflection on this case, the senior authors may revise future sternal replacements in patients with significant co-morbidities. Instead of the web design for ingrowth of bone, a solid implant will be considered, as this would mean that bony ingrowth is not required, and this burden of healing is removed.

\section{Acknowledgments}

Funding: None.

\section{Footnote}

Conflicts of Interest: All authors have completed the ICMJE uniform disclosure form (available at https://ccts. amegroups.com/article/view/10.21037/ccts.2020.02.01/ coif). The authors have no conflicts of interest to declare.

Ethical Statement: The authors are accountable for all aspects of the work in ensuring that questions related to the accuracy or integrity of any part of the work are appropriately investigated and resolved. All procedures performed in this study were in accordance with the ethical standards of the institutional and national research committees and with the Helsinki Declaration (as revised in 2013). Written informed consent was obtained from the patient for publication of this case report. A copy of the written consent is available for review by the editorial office of this journal.

Open Access Statement: This is an Open Access article distributed in accordance with the Creative Commons Attribution-NonCommercial-NoDerivs 4.0 International 
License (CC BY-NC-ND 4.0), which permits the noncommercial replication and distribution of the article with the strict proviso that no changes or edits are made and the original work is properly cited (including links to both the formal publication through the relevant DOI and the license). See: https://creativecommons.org/licenses/by-nc$\mathrm{nd} / 4.0 /$.

\section{References}

1. Abu-Omar Y, Kocher GJ, Bosco P, et al. European Association for Cardio-Thoracic Surgery expert consensus statement on the prevention and management of mediastinitis. Eur J Cardiothorac Surg 2017;51:10-29.

2. van Wingerden JJ, Ubbink DT, van der Horst CM, et al. Poststernotomy mediastinitis: a classification to initiate and evaluate reconstructive management based on evidence from a structured review. J Cardiothorac Surg 2014;9:179.

3. Bonesupport. Gentamicin release in vitro from setting

doi: $10.21037 /$ ccts.2020.02.01

Cite this article as: Trevis J, Dunning J, McVie J. Surgical reconstruction of the sternum following cardiac surgery complicated by mediastinitis-implantation of a custommade sternal implant: a case report. Curr Chall Thorac Surg 2020;2:31.
CERAMENT G paste. Data on file BONESUPPORT: Sweden report S009/2012.

4. Ennker IC, Ennker JC. Management of sternomediastinitis. HSR Proc Intensive Care Cardiovasc Anesth 2012;4:233-41.

5. Deniz H, Gokaslan G, Arslanoglu Y, et al. Treatment outcomes of postoperative mediastinitis in cardiac surgery; negative pressure wound therapy versus conventional treatment. J Cardiothorac Surg 2012;7:67.

6. Sanna S, Brandolini J, Pardolesi A, et al. Materials and techniques in chest wall reconstruction: a review. J Vis Surg 2017;3:95.

7. Pedersen TAL, Pilegaard HK. Reconstruction of the thorax with Ley Prosthesis after resection of the sternum. Ann Thorac Surg 2009;87:e31-3.

8. Kaláb M, Karkoška J, Kamínek M, et al. Reconstruction of massive post-sternotomy defects with allogeneic bone graft: four-year results and experience using the method. Interact Cardiovasc Thorac Surg 2016;22:305-13. 